Document downloaded from:

http://hdl.handle.net/10251/156327

This paper must be cited as:

Saez-Ferre, S.; Lopes, CW.; Simancas-Coloma, J.; Vidal Moya, JA.; Blasco Lanzuela, T.; Agostini, G.; Mínguez Espallargas, G.... (2019). Use of Alkylarsonium Directing Agents for the Synthesis and Study of Zeolites. Chemistry - A European Journal. 25(71):16390-16396. https://doi.org/10.1002/chem.201904043

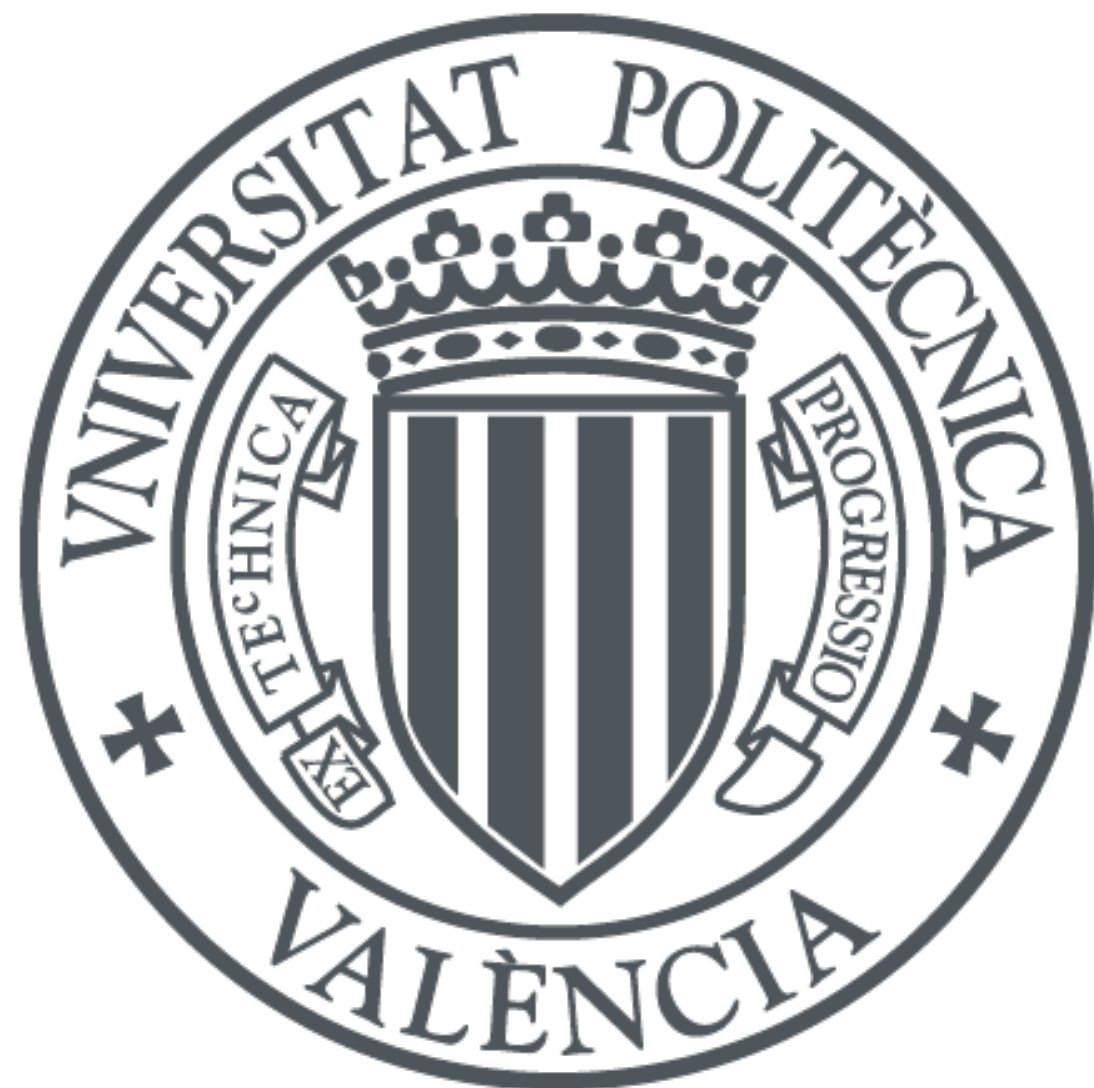

The final publication is available at

https://doi.org/10.1002/chem.201904043

Copyright John Wiley \& Sons

Additional Information

This is the peer reviewed version of the following article: Chem. Eur. J. 2019, 25, 16390 16396 , which has been published in final form at https://doi.org/10.1002/chem.201904043. This article may be used for non-commercial purposes in accordance with Wiley Terms and Conditions for Self-Archiving. 


\title{
Use of alkylarsonium directing agents for the synthesis and study of zeolites
}

Sara Sáez-Ferre, ${ }^{1}$ Christian W. Lopes, ${ }^{1,2}$ Jorge Simancas, ${ }^{1}$ Alejandro Vidal-Moya, ${ }^{1}$ Teresa Blasco, ${ }^{1}$ Giovanni Agostini, ${ }^{3}$ Guillermo Mínguez Espallargas, ${ }^{4}$ Jose L. Jordá, ${ }^{1 *}$ Fernando Rey ${ }^{1 *}$ and Pascual Oña-Burgos ${ }^{1 *}$

${ }^{1}$ Instituto de Tecnología Química (UPV-CSIC), Universitat Politècnica de València-Consejo Superior de Investigaciones Científicas, Avda. de los Naranjos s/n, 46022 Valencia, Spain.

${ }^{2}$ LRC - Institute of Chemistry, Universidade Federal do Rio Grande do Sul, 91501-970 Porto Alegre, Brazil.

3 ALBA Synchrotron Light Facility, Carrer de la Llum 2-26, 08290 Cerdanyola del Vallés, Barcelona, Spain.

${ }^{4}$ Instituto de Ciencia Molecular (ICMol), Universidad de Valencia, c/Catedrático José Beltrán, 2, 46980 Paterna, Spain.

*jjorda@itq.upv.es; frey@itq.upv.es; pasoabur@itq.upv.es

\begin{abstract}
Expanding the previously known family of onium (ammonium, phosphonium and sulfonium) organic structure-directing agents (OSDAs) for the synthesis of zeolite MFI, a new member, the arsonium cations, is used for the first time. The new group of tetraalkylarsonium cations has allowed synthesizing the zeolite ZSM-5 with several different chemical compositions, opening a route for the synthesis of zeolites with new series of OSDA. Moreover, the use of As replacing $\mathrm{N}$ in the OSDA allows introducing probe atoms that facilitate the study of these molecules by powder X-ray diffraction (PXRD), solid-state nuclear magnetic resonance (MAS-NMR) and Xray absorption spectroscopy (XAS). Finally, the influence of trivalent elements such as B, Al or Ga isomorphically replacing $\mathrm{Si}$ atoms in the framework structure and its interaction with the As species has been studied. We have proved the suitability of tetraalkylarsonium cation for carrying out crystallization of zeolites and also, the benefit of the presence of As atoms in the occluded OSDA, which allows its advanced characterization as well as the study of its evolution during OSDA removal by thermal treatments.
\end{abstract}




\section{Introduction}

Zeolites are crystalline microporous materials, mainly constituted by silicon oxide where the $\mathrm{Si}$ atom can be isomorphically replaced by other elements like Al, B, Ga, Ti, Ge or Sn. The welldefined size and distribution of the structural channels of each different zeolitic framework type together with the chemical composition confer to these materials multiple applications in processes such as gas adsorption and separation, catalysis and encapsulation or controlled release of molecules, among others, some of them with industrial applications. ${ }^{[1-9]}$ This is the driving force for studying novel methods for the preparation of new zeolitic structures, since it would give the possibility of obtaining tailored materials with the most appropriate channel system for each specific application. To date, up to 234 fully ordered as well as 11 partially disordered different zeolitic structures have been accepted by the International Zeolite Association, ${ }^{[10]}$ and this number is constantly increasing.

Organic structure-directing agents (OSDAs) are fundamental tools for the preparation of zeolitic structures, ${ }^{[11-13]}$ but their interaction with the zeolitic frameworks is unclear due to the difficulty in their localization in the crystalline structure. This is currently a challenge that usually requires the use of synchrotron radiation or the characterization through single crystal X-ray diffraction, very uncommon for zeolites. ${ }^{[14-16]}$ Therefore, the development of straightforward and accessible methodologies to allow the structural elucidation using laboratory X-ray powder diffraction techniques is of clear interest.

In this sense, the incorporation of heavy atoms with high electron densities and large scattering factors could be of interest for the location of OSDA moieties placed inside of zeolite voids due to the introduction of a large electronic contrast. Phosphorous containing OSDAs have been used for directing the crystallization of a number of zeolites, where $\mathrm{N}$ atoms of tetraalkylammonium OSDAs were substituted by $\mathrm{P}$ atoms yielding to tetraalkylphosphonium analogues. ${ }^{[17-23]}$ This approach has produced a series of new zeolites, and the occluded organic cations were characterized by ${ }^{31} \mathrm{P}-\mathrm{MAS}-\mathrm{NMR}$ spectroscopy. Ever more, the use of sulfonium compounds have recently proved to be also successful for the synthesis of some zeolitic materials. ${ }^{[24-25]}$ However, 
the electron density of the $\mathrm{P}$ or $\mathrm{S}$ atoms was insufficient for easily locating the organic template using lab data.

Up to now, all this development of new OSDAs, based almost exclusively on ammonium, phosphonium or even sulfonium cations, has opened the synthesis of new zeolite structures. ${ }^{[26]}$ Herein, we describe the use of a new family of OSDAs, the tetraalkylarsonium cations for the synthesis of the zeolite ZSM-5 (IZA code MFI), which is a proof of concept of the ability of arsonium cations as OSDAs for the synthesis of zeolites. Furthermore, we show that the use of arsenic plays an essential role in the study of the OSDA entrapped in the zeolite, as its higher electronic density respect to phosphorus, sulphur or nitrogen allows its facile localization by laboratory powder X-ray diffraction, as well as allows the use of ${ }^{75}$ As MAS-NMR and XAS at the As K-edge to analyse the location and properties of the molecule. In this sense, the As atom can be considered as a probe inside the OSDA molecule to enable for its study. Additionally, the removal of the OSDA by calcination and/or reduction has been also studied.

\section{Results and discussion}

For this work, three tetraalkylarsonium cations have been synthesized: triethylmethylarsonium (OSDA-1), tetraethylarsonium (OSDA-2) and 1,4-bis-(triethylarsonium)butane (OSDA-3) cations as described in Scheme S1. The synthesis and characterization of all these cations is described in the Electronic Supplementary Information (ESI). Moreover, a safe handling is required to work with triethyl-arsine precursor in the synthesis of arsonium cation and volatile arsenic containing compounds formed during OSDA removal by thermal treatments, which is described in detail in the ESI. In this point should be indicated that arsonium cations themselves are really stable and with low toxicity. ${ }^{[27]}$

Zeolite MFI was obtained as single phase using OSDA-2 as described in the ESI (Figures S11 and S12). The samples show the typical coffin shape of MFI materials observed for high silica materials (Figures S21-S24). ${ }^{[28]}$ This result is in consonant to the observed crystallization of zeolite MFI using the analogous tetraethylammonium and tetraethylphosphonium cations as OSDAs. 
Interestingly, OSDA-1 and OSDA-3 gave similar results, although longer crystallization times were required (typically 21 days), suggesting that the common triethylarsonium moiety is driving the crystallization to MFI zeolitic materials.

Moreover, replacing part of the silica source with $\mathrm{GeO}_{2}, \mathrm{Al}_{2} \mathrm{O}_{3}, \mathrm{Ga}\left(\mathrm{NO}_{3}\right)_{3}$ or $\mathrm{H}_{3} \mathrm{BO}_{3}$ in the crystallization media allows the isomorphic incorporation of those elements into the zeolitic framework when the OSDA-2 is used as structure directing agent (Figures S11 and S12). However, this substitution does not occur when any of the other two OSDAs were used and only amorphous solids were recovered under similar conditions that pure silica MFI (Figure S11b).

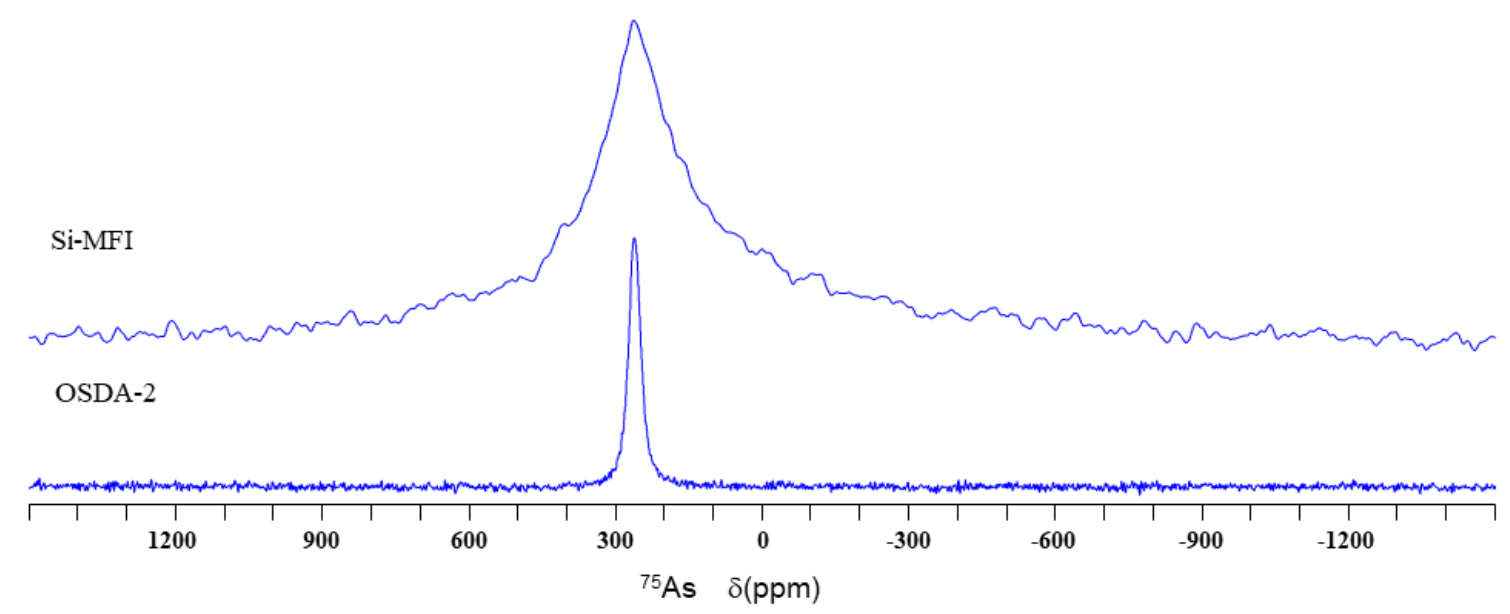

Figure 1: ${ }^{75}$ As MAS NMR for the OSDA-2 (a) and as-prepared Si-MFI (b).

After performing the synthesis, the structural integrity of the OSDA inside the pure as-made silica zeolite was checked. Chemical analyses of the as-made sample revealed a C/As ratio was equal to 8 , very close to theoretical value of the OSDA-2 (Table S2). Also, the chemical shift of the signal appearing at $250 \mathrm{ppm}$ in the ${ }^{75} \mathrm{As}$ MAS-NMR spectrum of the OSDA-2-containing zeolite Si-MFI is almost identical to that of the free OSDA (Figure 1). The larger broadening for the OSDA-2@MFI material can be associated to an increase of the quadrupole coupling constant of the ${ }^{75} \mathrm{As}(\mathrm{I}=3 / 2)$. The confinement of the OSDA-2 within the zeolite channels decreases the mobility of the cation and enhances the electric field gradients of its surrounding. In addition, the integrity of the OSDA during the synthesis conditions was carefully studied by ${ }^{1} \mathrm{H}$ NMR. In this 
sense, the ${ }^{1} \mathrm{H}$ NMR spectrum was acquired for the mother liquor and compared with ${ }^{1} \mathrm{H}$ NMR spectrum for the free OSDA in solution (Figure S14). Comparison of both spectra confirms again that the OSDA is stable under synthesis conditions. Since, the OSDA-2 remains unchanged after MFI crystallization, its exact location inside of the zeolite structure was obtained employing lab X-ray data. In order to do so, the orthorhombic structure of MFI of the as-made material obtained using tetrapropylammonium as OSDA, ${ }^{[29]}$ excluding the organic component, was used as the starting point. Then, a difference Fourier map was calculated using the programs FullProf and GFourier. This map allowed easily determining the location of the arsenic atoms, due to their high electron density, which reveals the otherwise elusive position of the molecule. Moreover, the approximated location of the $4 \mathrm{C}$ atoms connected to As (Figures 2a and S13) has been easily obtained. The 4 remaining $\mathrm{C}$ atoms where located close to the previous ones, and geometrical restraints were imposed prior to the refinement. The used restraints were obtained from the singlecrystal X-ray diffraction analysis of the pure OSDA (Figure S10 and Table S1). Last, a fluorine ion was placed close to the 4 ring, as it has been widely described that $\mathrm{F}^{-}$species are located close to those units. ${ }^{[30]}$ The lab XRPD pattern was refined using the program FullProf, obtaining good residual values, as well as a good matching between the experimental and calculated patterns (Figure 2b).
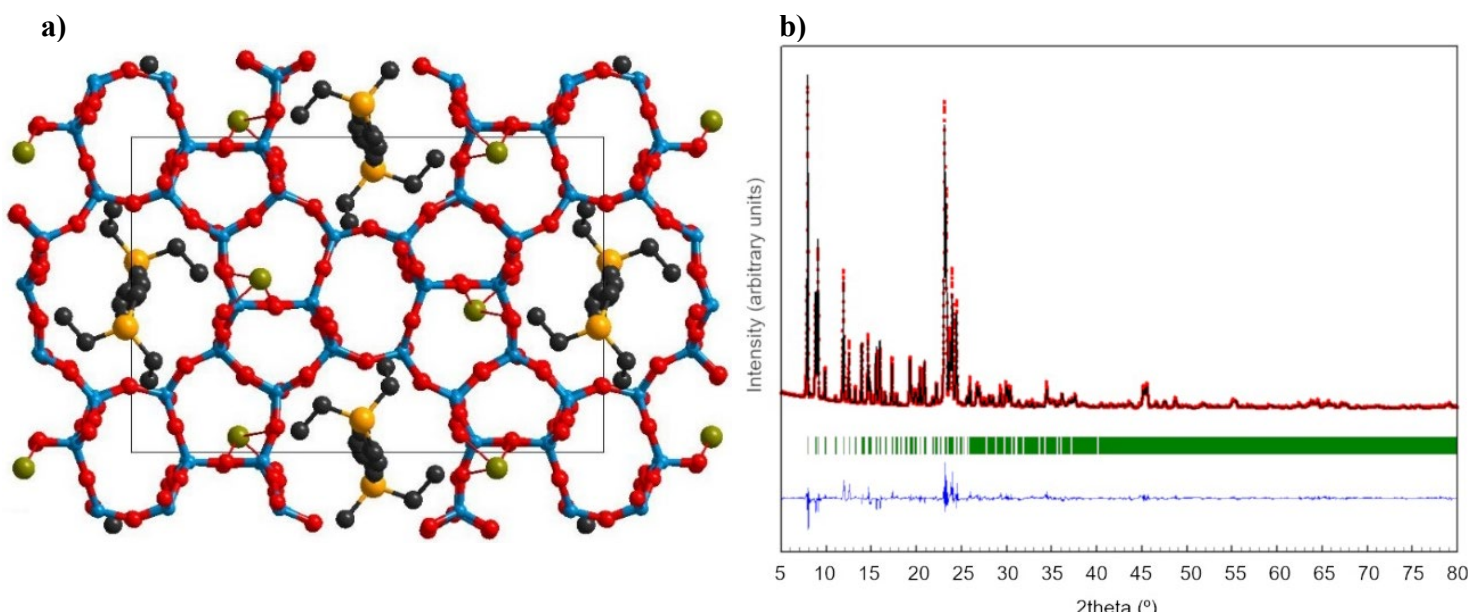

Figure 2. a) OSDA-2 localized inside the zeolite Si-MFI (Si blue, O red, As gold, C black and F green); b) Rietveld refinement of the X-ray diffraction pattern of as-made MFI. Observed (red circles) and calculated (black line) XRPD patterns, and the corresponding difference profile (bottom, blue line). The blue short ticks marks below the pattern give the positions of the Bragg reflections. Residual values $\left(R_{w p}=14.1, R_{\exp }=2.36, R_{B}=7.70, R_{F}=5.26\right)$. 
One interesting fact is that the location of the OSDA-2 in MFI is very similar for both tetraethylarsonium, herein determined by lab XRPD, and tetrapropylammonium, previously found by single-crystal X-ray diffraction. ${ }^{[29]}$ Therefore, both organic cations, based on arsenic and nitrogen atoms respectively, seem to have an analogous interaction with the zeolitic framework. In addition, the location of $\mathrm{F}^{-}$is in good agreement to that suggested by using ${ }^{19} \mathrm{~F}-\mathrm{MAS}-\mathrm{NMR}$ data (Figure S15), where a main F-NMR signal was observed at $-67 \mathrm{ppm} .{ }^{[30]}$

Further analysis of the as-made sample was done by X-ray absorption spectroscopy. In this sense, we must take into account that, although alkylammonium, and less frequently phosphonium cations, are by far the most commonly used molecules as OSDA, the X-ray absorption edges of $\mathrm{N}$ or $\mathrm{P}$ atoms are at very low energies and their XAS spectra cannot be easily measured. Distinctly here, we can take profit of the presence of a heavy atom, like As, in the OSDA-2 cation and perform its characterization by XAS at As K-edge $(11867 \mathrm{eV})$. So, the organic cation containing As, either in its iodide form or entrapped inside the MFI channels after hydrothermal zeolite synthesis, was investigated by XAS allowing to further probed the stability of As species during the hydrothermal synthesis.
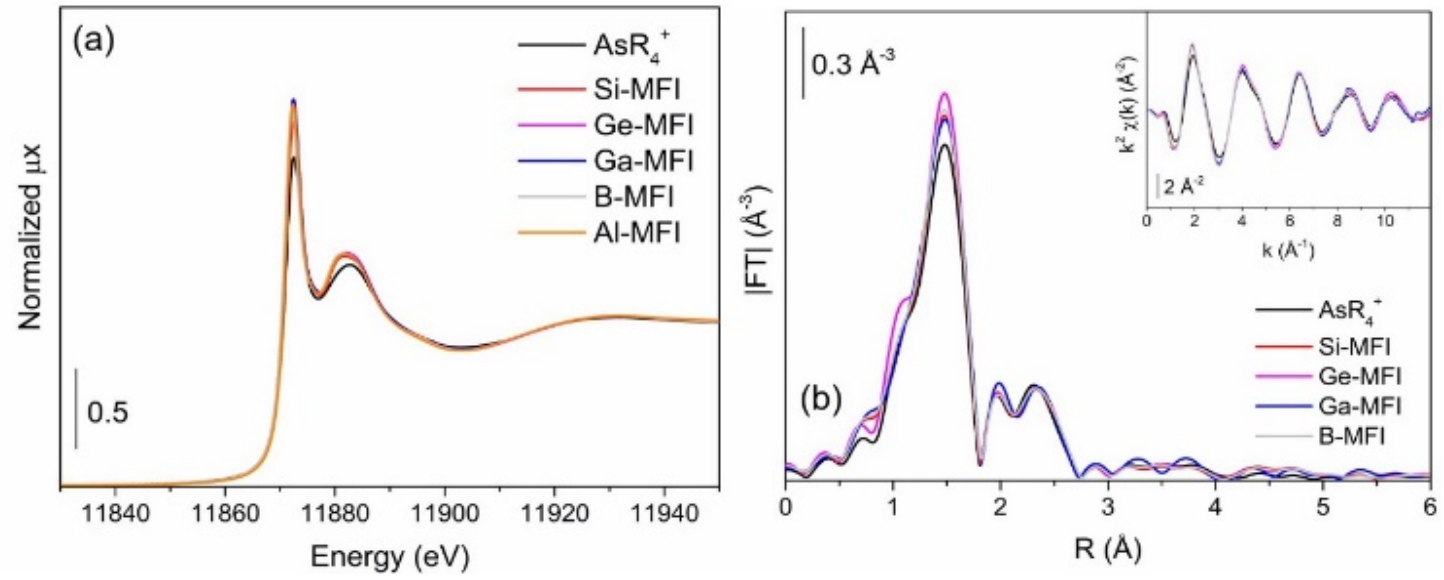

Figure 3. Normalized XANES spectra at As K-edge (a) and $|\mathrm{FT}|$ of $\mathrm{k}^{2}$-weighted $\chi(\mathrm{k})$ functions of crystallized $\mathrm{AsR}_{4}{ }^{+}$ and as-synthesized As@ zeolites MFI (b). Inset of (b) represents the $\mathrm{k}^{2}$-weighted $\chi(\mathrm{k})$ functions of displayed data.

The XANES region of the OSDA-2 in its iodide form and those entrapped inside of the zeolite channels are nearly identical (Figure 3a) indicating that the As-containing OSDA remains intact inside all the zeolites obtained in this study, in good agreement with the ${ }^{75}$ As-NMR (Figure 1) and chemical analyses (Table S2). The fitting of the EXAFS data of the pure OSDA-2 with As- 
$\mathrm{C}$ contribution gives a coordination number around 4 (Figure $3 \mathrm{~b}$ and Table1); also very close to that obtained for the EXAFS spectrum of the as-synthesized material, with distances of $1.932 \pm$ $0.005 \AA$. This is in good agreement with theoretical and also crystallographic structure of the $[\mathrm{OSDA}-2]^{+} \mathrm{I}^{-}$salt, solved by single-crystal XRD. The as-made zeolites show similar edge position and features beyond the edge of the OSDA-2, which confirms that organic template species are stable during the zeolites syntheses. At this point, the OSDAs entrapped in the MFI materials containing different heteroatoms $(\mathrm{B}, \mathrm{Al}, \mathrm{Ga}$ and $\mathrm{Ge})$ were also studied by means of XAS. The corresponding XANES and EXAFS spectra are shown in Figure 3 and fitted EXAFS parameters are reported in Table 1.

Table 1. Summary of optimized parameters by fitting EXAFS data of crystallized AsR ${ }^{+}$and as-synthesized As@ MFI materials ${ }^{\mathrm{a}}$

\begin{tabular}{|c|c|c|c|c|c|}
\hline Sample & $\mathbf{N}_{\text {As-C }}$ & $\mathbf{R}_{\text {As-C }}(\AA)$ & $\sigma^{2}\left(\AA^{2}\right)$ & $\Delta E_{0}(e V)$ & R-factor \\
\hline OSDA-2 & $4.0 \pm 0.2$ & $1.932 \pm 0.005$ & \multirow{5}{*}{$0.0018 \pm 0.0004$} & \multirow{5}{*}{$7.7 \pm 0.3$} & 0.00877 \\
\hline Si-MFI & $4.5 \pm 0.2$ & $1.936 \pm 0.004$ & & & 0.00653 \\
\hline Ge-MFI & $4.8 \pm 0.2$ & $1.937 \pm 0.004$ & & & 0.00909 \\
\hline Ga-MFI & $4.5 \pm 0.4$ & $1.938 \pm 0.009$ & & & 0.01567 \\
\hline B-MFI & $4.5 \pm 0.2$ & $1.940 \pm 0.003$ & & & 0.00634 \\
\hline
\end{tabular}

${ }^{a} \mathrm{~A}$ simultaneous fit of the spectra was adopted, fixing the $\sigma^{2}$ and $\Delta \mathrm{E}_{0}$ values; the fits were performed on the first coordination shell $(\Delta \mathrm{R}=1.0-2.0 \AA)$ over FT of the $\mathrm{k}^{1} \mathrm{k}^{2} \mathrm{k}^{3}$-weighted $\chi(\mathrm{k})$ functions performed in the $\Delta \mathrm{k}=2.0-12.0 \AA^{-1}$ interval, resulting into a number of independent parameters of $2 \Delta \mathrm{R} \Delta \mathrm{k} / \pi=31.0 . \mathrm{S}_{0}^{2}=0.86$.

The MFI materials were calcined under dry air at $650{ }^{\circ} \mathrm{C}$, observing by XRD that all of them are stable after calcination (Figure S27). However, there are clear differences in the final As content in the calcined materials depending on the presence of trivalent heteroatoms isomorphically incorporated into the MFI framework. Indeed, after calcination, no As is observed in the samples containing only tetravalent (i.e. Si and Ge) atoms. While, around $50 \%$ of the initial As content is still present after calcination for those MFI-zeolites containing trivalent elements like $\mathrm{Ga}, \mathrm{Al}$ or B. In these later cases, the molar ratio trivalent element to As (M/As) is close to 1 (see Table S3). These results strongly suggest that the arsenic atoms that are not interacting with trivalent element evolve out of the zeolite during calcination, while the arsenic that interacts with T(III) elements is retained inside the zeolite. So thus, the presence of trivalent elements plays a pivotal role to keep the arsenic inside the porous network of the zeolite. However, the ${ }^{75}$ As MAS-NMR spectra of the calcined samples do not show any resonance, despite the as-made materials give the broad 
bands characteristic of the OSDA inside the zeolite (Figure S28) and chemical analyses indicate that significant amount of As remains within the zeolite upon calcination. The absence of Assignals is due to the formation of low-symmetry species with very large quadrupole couplings giving so broad signals that become undetectable by conventional NMR experiments. ${ }^{[31]}$

When the as-made sample is treated under hydrogen at high temperature instead of by calcination (Figure S27), As atoms are almost removed independently of the chemical composition of the framework (see Table S3).

Additionally, the isomorphic incorporation of the trivalent $\mathrm{Al}$ and $\mathrm{Ga}$ cations into the framework positions was further confirmed by ${ }^{27} \mathrm{Al}$ and ${ }^{71} \mathrm{Ga}$ MAS-NMR, respectively. The ${ }^{27} \mathrm{Al}$ MAS-NMR spectra of as-synthesized and calcined MFI samples are shown in ESI (Figure S29). The ${ }^{27} \mathrm{Al}$ MAS-NMR spectra of the as-made and calcined samples show a main signal centered at $52 \mathrm{ppm}$, which is assigned to aluminum in tetrahedral sites. ${ }^{[32]}$ Also, a residual resonance is observed at 0 ppm, which are typically attributed to the presence of extra-framework aluminum in the zeolite. ${ }^{[32]}$ For the Ga-containing material, resonances at $152 \mathrm{ppm}$ in the ${ }^{71} \mathrm{Ga}$ MAS-NMR spectra of the assynthesized and calcined samples were detected (Figure S30) and assigned to gallium in tetrahedral coordination, corresponding to framework positions. ${ }^{[33]}$

The evolution of the As species within MFI structure during calcination in air was 'in-situ' studied on the Ga-MFI sample. The raw data of the XANES region indicate that by increasing the temperature (Figure 4), the characteristic features present in the spectrum of OSDA-2 are maintained until $\sim 500{ }^{\circ} \mathrm{C}$, where the oscillation centered at $11882 \mathrm{eV}$ starts to disappear, indicating that the local structure around As atom was modified above this temperature as a consequence of the OSDA decomposition (in agreement with TGA data shown in Figures S16S20). 


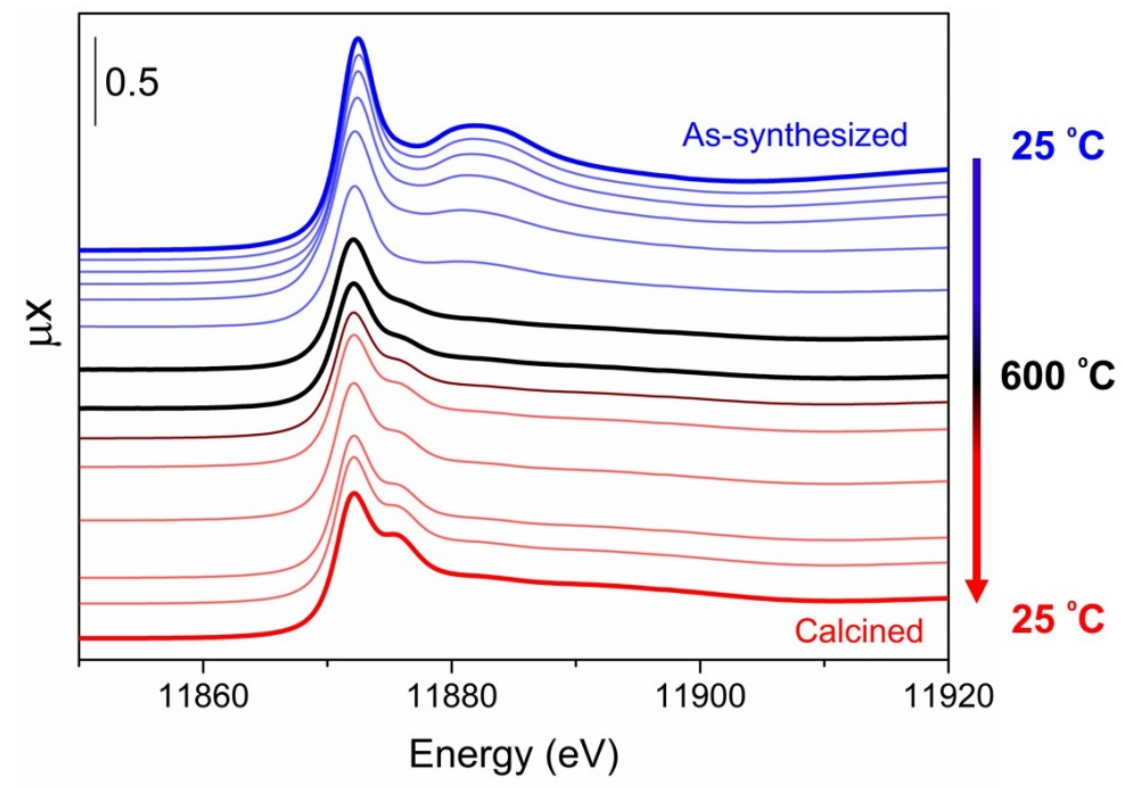

Figure 4. Raw XANES spectra at As K-edge of Ga-MFI zeolite during calcination: the shoulder at $11882 \mathrm{eV}$ characteristic of the OSDA is lost throughout the thermal treatment.

When the temperature reaches $600{ }^{\circ} \mathrm{C}$, this feature gets flattened and a shoulder at $11875 \mathrm{eV}$ grown up as far as the temperature decreases to $25^{\circ} \mathrm{C}$. This feature could be attributed to the oxidation of $\mathrm{As}^{3+}$ to $\mathrm{As}^{5+}$, however, the edge position is maintained as that characteristic for $\mathrm{As}^{3+} \cdot{ }^{[34]}$ Moreover, $33 \%$ of As was released during calcination, most likely as volatile $\mathrm{AsR}_{3}$ which is formed during this thermal treatment (Table S4). This value is in reasonable agreement with chemical analysis of Ga-MFI sample submitted to calcination in the laboratory. A similar behavior has been observed by our group for phosphorous containing cations, which were transformed into phosphate-like species upon calcination. ${ }^{[23]}$

The XANES spectra of the As-Ga-MFI after thermal treatments under hydrogen at $600{ }^{\circ} \mathrm{C}$ exhibit a similar feature, but with some differences in terms of the amount of As released. When the fresh Ga-MFI sample is directly reduced in $\mathrm{H}_{2}$, only a small fraction of As remains (approx. 5\% - Table S4) at the end of thermal treatments since the presence of $\mathrm{H}_{2}$ facilitates the formation of volatile $\mathrm{AsR}_{3}$ species. On the other hand, the subsequent reduction of the calcined As-Ga-MFI material shows that around $20 \%$ of the As atoms is still present in the zeolite at the end of the subsequent reduction, demonstrating that calcination treatment is able to stabilize most of the As atoms against reduction in the zeolite. 
In order to investigate the difference of As species formed on Ga-MFI depending on the thermal treatment, the samples were 'in-situ' studied during calcination, reduction and calcinationreduction treatments. The normalized XANES spectra of these samples are compared with those of standard As compounds in Figure 5a. The XANES spectra for samples after any thermal treatment are rather similar regardless the heating procedure or atmosphere employed, with same edge position (ca. $11870 \mathrm{eV}$ ) as for OSDA-2, indicating that the formal oxidation state is maintained as $\mathrm{As}^{3+}$. However, as stated above, the oscillation at $11882 \mathrm{eV}$ present for OSDA-2 is lost upon any of the studied thermal treatments, demonstrating that the organic compound decomposes at the end of the experiments. Furthermore, the shape of the spectra does not resemble those of arsenic oxides (i.e. $\mathrm{As}_{2} \mathrm{O}_{3}$ and $\mathrm{As}_{2} \mathrm{O}_{5}$ ) or that for $\mathrm{As}^{0}$, indicating that different species are formed in the zeolites. While, the shape of XANES spectra displayed here is compatible with those found for $\mathrm{As}^{3+}$ cations adsorbed on surfaces in experiments of wastewater remediation, consisting in arsenic inner(outer) complexes. ${ }^{[35-37]}$ Aiming to obtain more detailed information on the local structure of these As-based species, the EXAFS region was analyzed (Figure 5b). Only a first-shell As-O contribution is seen in most $|\mathrm{FT}|$ for thermally treated samples, pointing out that As atoms are involved in a highly dispersed or disordered arrangement. Furthermore, different intensities of As-O contribution can be observed, which indicates that As atoms are differently coordinated or display unlike static disorder (different Debye-Waller factors). For disentangle these questions, the quantitative information was extracted by treating the EXAFS data, which is gathered in the ESI (Table S5 and Figure S31 for fitted data). The three thermally treated samples show a coordination number around As of 3.5, with As-O distances of $\sim 1.75 \AA$ (within errors). These values are in agreement with those found in literature for As bounded to the surface of adsorbents (inner-shell complexes). ${ }^{[35-38]}$ However, the samples which undergone a calcination process (calcined and calcined-reduced) show similar Debye-Waller factors, while the reduced one showed significant higher value, suggesting that As atoms are in more disordered local environment, which can explain its lower As-O first-shell contribution intensity at $|\mathrm{FT}|$ with respect to those that undergone a calcination process. The meaningful $\Delta \mathrm{E}_{0}$ value is also in agreement with those found in literature for As-based species studied by XAS. ${ }^{[38-39]}$ 

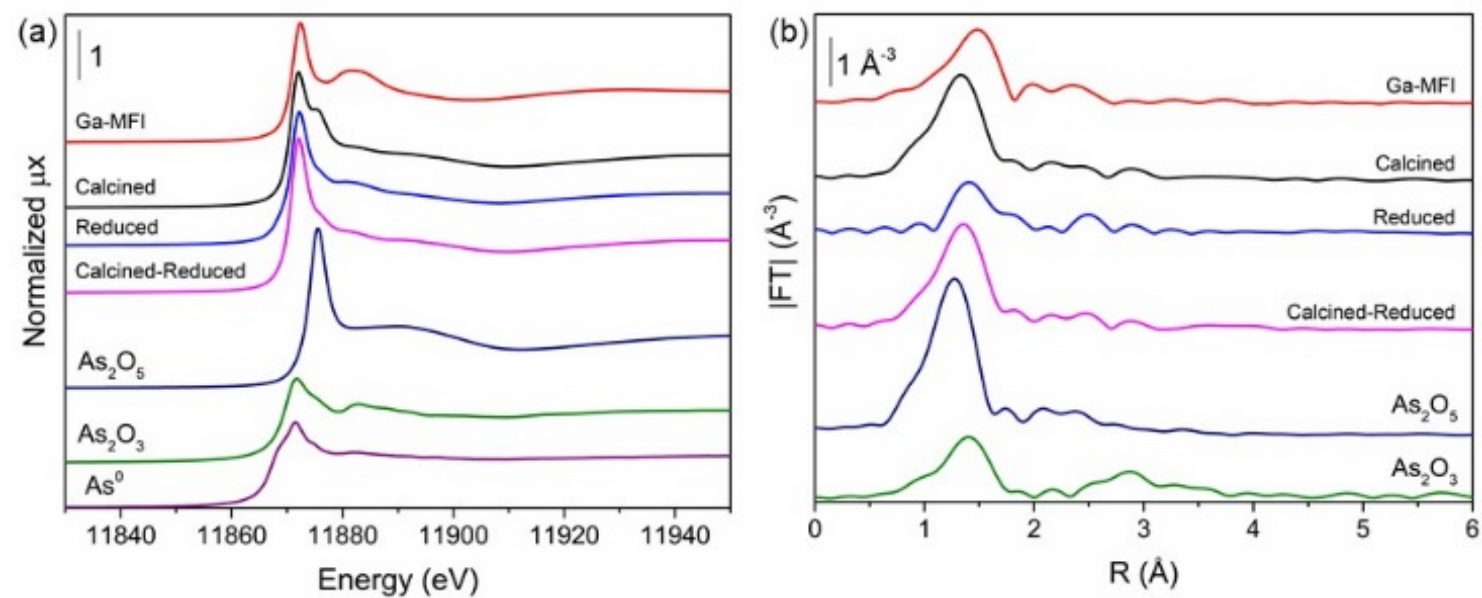

Figure 5. Normalized XANES spectra at As K-edge (a) and $|\mathrm{FT}|$ of $\mathrm{k}^{2}$-weighted $\chi(\mathrm{k})$ functions of Ga-MFI before, after thermal treatments and As-based standards (b).

Finally, the nature of the Ga species present in the zeolitic framework was also studied by XAS at Ga K-edge. The XANES spectrum of as-synthesized Ga-MFI (Figures S32-S33) is similar to that reported for Ga-substituted zeolite Beta, ${ }^{[40]}$ with the absorption edge positioned at $10372 \mathrm{eV}$, typical of $\mathrm{Ga}^{3+}$. Moreover, no indicia of $\mathrm{Ga}_{2} \mathrm{O}_{3}$ phase can be observed in the XAS spectrum of the as-made material. By submitting the samples to the different thermal treatments (calcination, reduction and calcination-reduction) the Ga atoms remain mostly tetrahedrally coordinated, with exception of the sample after calcination-reduction. In this case, a shoulder at $10367 \mathrm{eV}$ appeared, showing that a small portion of $\mathrm{Ga}$ atoms are reduced after subsequent calcination-reduction treatments, which can be interpreted as a migration of Ga from framework to extra-framework positions. Isolating the EXAFS region of the spectra gives rise to the presence of a predominant first-shell Ga-O contribution in case of Ga-MFI before and after treatments, which is rather different respect to that of $\mathrm{Ga}_{2} \mathrm{O}_{3}$ (Figure S32 and Table $\mathrm{S} 6$ for fitted data). A decrease in the intensity of this first-shell contribution is observed after thermal treatments, as already previously reported. ${ }^{[4]}$ The as-synthesized Ga-MFI still containing the OSDA-2 gives a coordination number of 4 with Ga-O distances of $\sim 1.78 \AA$ (within errors, see Table S6), in agreement with data reported in the literature. ${ }^{[40,42]}$ These results confirm that Ga atoms are tetrahedrally coordinated within MFI framework in excellent agreement with previously discussed ${ }^{71} \mathrm{Ga}-\mathrm{MAS}-\mathrm{NMR}$ results. 


\section{Conclusions}

In summary, this study has achieved several milestones, which could become in a breakthrough in the synthesis of zeolites. First, we have probed that alkylarsonium can be used as efficient OSDAs for synthesis of zeolite MFI. This achievement could open the possibility for obtaining novel zeolites as previously occurred with phosphonium organocations with respect to N-based OSDAs. ${ }^{[17-23]}$ Also, we have shown that Arsenic atoms included in OSDAs can be used as useful probe moieties for understanding the behavior of occluded structure directing agents during the thermal treatments for zeolite activation. The high electron density of arsenic atoms is extremely helpful in order to localize the OSDA in the zeolitic structure by powder X-ray diffraction, and the location of the OSDA molecule in the MFI structure is analogous to that described for the ammonium derivate. Additionally, the presence of As in the OSDA allows the study of the OSDA and of the As species trapped in the zeolite after the calcination by ${ }^{75}$ As MAS-NMR and XAS at the As K-edge. Finally, it has been evidenced that arseniate-like species interact with the trivalent Ga sites at the framework during the thermal treatments of the samples. Then, we have shown that arsenic atoms are useful for characterization and evolution of OSDA entrapped in the zeolitic voids.

\section{Methods}

Detailed information on the condition of samples preparation and of data acquisition and analysis for the different techniques are provided in the Electronic Supplementary information.

\section{Data availability}

Crystallographic data for the OSDA-2 structure reported in this Article has been deposited at the Cambridge Crystallographic Data Centre, under deposition no. CCDC 1910863. Copies of the data can be obtained free of charge from www.ccdc.cam.ac.uk/structures/.

All other data supporting the results are available within the Article and its Electronic Supplementary Information, or from the corresponding authors upon reasonable request.

\section{Acknowledgements}


Program Severo Ochoa SEV-2016-0683 is gratefully acknowledged. S.S-F. thanks MEC for his Severo Ochoa Grant SPV-2013-067884, P. O-B. thanks MEC for his Ramón y Cajal contract RYC-2014-16620. Authors thank the financial support by the Spanish Government (RTI2018096399-A-I00 and RTI2018-101784-B-I00). The Electron Microscopy Service of the UPV is acknowledged for their help in sample characterization. We gratefully acknowledge ESRF synchrotron for allocating beamtime (proposal CH-5193), the Italian CRG beam-line at ESRF (LISA-BM08) and Alessandro Puri for the help and technical support during our experiment. C. W. Lopes (Science without Frontiers - Process no. 13191/13-6) thanks CAPES for a predoctoral fellowship.

\section{Keywords}

Organic Structural Directing Agents; alkylarsonium; zeolite synthesis and characterization.

\section{Competing interests}

The authors declare no competing interests.

\section{Additional information}

Supplementary information is available for this paper at the web page of Chemistry-A European Journal in Wiley-VCH.

\section{References}

[1] J. Sun, C. Bonneau, A. Cantin, A. Corma, M. J. Diaz-Cabañas, M. Moliner, D. Zhang, M. Li, and X. Zou, Nature, 2009, 458, 1154-1157.

[2] J. Jiang, J. Yu, and A. Corma, Angew. Chem. Int. Ed. 2010, 49, 3120-3145.

[3] A. Corma, J. Catal. 2003, 216, 298-312.

[4] M. E. Davis, Nature, 2002, 417, 813-821. 
[5] R. M. Shayib, N. C. George, R. Seshadri, A. W. Burton, L. S. I. Zones, and B. F. Chmelka, J. Am. Chem. Soc. 2011, 133, 18728-18741.

[6] Z. Wang, J. Yu, and R. Xu, Chem. Soc. Rev. 2012, 41, 1729-1749.

[7] A. Corma, and M. E. Davis, ChemPhysChem, 2004, 5, 304-313.

[8] S. I. Zones, Micropor. Mesopor. Mater. 2011, 144, 1-8.

[9] G. Bellussi, A. Carati, and R. Millini, In Zeolites and Catalysis: Synthesis, Reactions and Applications. J. Cejka, A. Corma, and S. I. Zones, Eds. (Wiley-VCH: Weinheim, Germany, 2010; Vol. 2, p 449).

[10] International Zeolite Association Website. http://www.iza-online.org/ (accessed October 4, 2018).

[11] A. W. Burton, and S. I. Zones, Stud. Surf. Sci. Catal. 2007, 168, 137-179.

[12] A. W. Burton, S. I. Zones, and S. Elomari, Colloid Interface Sci. 2005, 10, 211-219.

[13] S. I. Zones, Y. Nakagawa, G. S. Lee, C. Y. Chen, and L. T. Yuen, Micropor. Mesopor. Mater. 1998, 21, 199-211.

[14] A. B. Pinar, L. B. McCusker, C. Baerlocher, S.-J. Hwang, D. Xie, A. I. Benin, and S. I. Zones, New J. Chem. 2016, 40, 4160-4166.

[15] S. Smeets, L. B. McCusker, C. Baerlocher, S. Elomari, D. Xie, and S. I. Zones, J. Am. Chem. Soc. 2016, 138, 7099-7106.

[16] S. Smeets, L. B. McCusker, C. Baerlocher, D. Xie, C.-Y. Chen, and S. I. Zones, J. Am. Chem. Soc. 2015, 137, 2015-2020.

[17] D. L. Dorset, G. J. Kennedy, K. G. Strohmaier, M. J. Diaz-Cabanas, F. Rey, and A. Corma, J. Am. Chem. Soc. 2006, 128, 8862-8867.

[18] A. Corma, M. J. Diaz-Cabanas, J. L. Jorda, F. Rey, G. Sastre, and K. G. Strohmaier, J. Am. Chem. Soc. 2008, 130, 16482-16483. 
[19] A. Corma, M. J. Díaz-Cabañas, J. Jiang, M. Afeworki, D. L. Dorset, S. L. Soled, and K. G. Strohmaier, Proc. Natl. Acad. Sci. U.S.A. 2010, 107, 13997-14002.

[20] D. L. Dorset, K. G. Strohmaier, C. E. Kliewer, A. Corma, M. J. Díaz-Cabañas, F. Rey, and C. J. Gilmore, Chem. Mater. 2008, 20, 5325-5331.

[21] R. Simancas, D. Dari, N. Velamazan, M. T. Navarro, A. Cantin, J. L. Jorda, G. Sastre, A. Corma, and F. Rey, Science, 2010, 330, 1219-1222.

[22] M. Hernández Rodríguez, J. L. Jorda, F. Rey, and A. Corma, J. Am. Chem. Soc. 2012, 134, 13232-13235.

[23] J. Simancas, R. Simancas, P. J. Bereciartua, J. L. Jorda, F. Rey, A. Corma, S. Nicolopoulos, P. Pratim-Das, M. Gemmi, and E. Mugnaioli, J. Am. Chem. Soc. 2016, $138,10116-10119$.

[24] C. Jo, S. Lee, S. J. Cho, and R. Ryoo, Angew. Chem. Int. Ed. 2015, 54, 12805-12808.

[25] S. Lee, C. Jo, H. Park, J. Kim, and R. Ryoo, Micropor. Mesopor. Mat. 2019, 280, $75-81$.

[26] F. Rey, and J. Simancas, Beyond Nitrogen OSDAs, in: L. Gómez-Hortigüela (Ed.) Insights into the Chemistry of Organic Structure-Directing Agents in the Synthesis of Zeolitic Materials, (Springer International Publishing, Cham, 2018, pp. 103-138).

[27] D. Fattorini, A. Notti, and F. Regoli, Chem. Ecol. 2006, 22, 405-414.

[28] G. Bonilla, I. Díaz, M. Tsapatsis, H. K. Jeong, Y. Lee, and D. G. Vlachos, Chem. Mater. 2004, 16, 5697-5705.

[29] H. van Koningsveld, H. van Bekkum, and J.C. Jansen, Acta Crystallogr. 1987, B43, 127-132.

[30] C. A. Fyfe, D. H. Brouwer, A. R. Lewis, and J. M. Chézeau, J. Am. Chem. Soc. 2001, $123,6882-6891$.

[31] G. Balimann, and P. S. Pregosin, J. Magn. Reson. 1977, 26, 283-289. 
[32] J. Klinowski, Chem. Rev. 1991, 91, 1459-1479.

[33] C. R. Bayense, J. H. C. van Hooff, A. P. M. Kentgens, J. W. de Haan, and L. J. M. van de Ven, J. Chem. Soc., Chem. Commun. 1989, 1992-1993.

[34] J. Canche-Tello, M. C. Vargas, J. Hernandez-Cobos, I. Ortega-Blake, A. Leclercq,

P. L. Solari, J. Lezama-Pacheco, C. Den Auwer, and J. M. de Leon, J. Phys. Chem. A, 2015, 119, 2829-2833.

[35] Y. Arai, E. J. Elzinga, D. L. Sparks, J. Colloid Interface Sci. 2001, 235, 80-88.

[36] M. L. Farquhar, J. M. Charnock, F. R. Livens, and D. J. Vaughan, Environ. Sci. Technol. 2002, 36, 1757-1762.

[37] G. Morin, G. Ona-Nguema, Y. Wang, N. Menguy, F. Juillot, O. Proux, F. Guyot, G. Calas, and G. E. Brown Jr, Environ. Sci. Technol. 2008, 42, 2361-2366.

[38] A. Ramirez-Solis, R. Mukopadhyay, B. P. Rosen, and T. L. Stemmler, Inorg. Chem. 2004, 43, 2954-2959.

[39] J. Canche-Tello, M. C. Vargas, J. Hernandez-Cobos, I. Ortega-Blake, A. Leclercq, P. L. Solari, C. Den Auwer, and J. Mustre de Leon, J. Phys. Chem. A, 2014, 118, 1096710973.

[40] C. Prieto, T. Blasco, M. Camblor, and J. Pérez-Pariente, J. Mater. Chem. 2000, 10, $1383-1387$.

[41] C. Lamberti, G. Turnes Palomino, S. Bordiga, A. Zecchina, G. Spanò, and C. Otero Areán, Catal. Lett. 1999, 63, 213-216.

[42] S. A. Axon, K. Huddersman, and J. Klinowski, Chem. Phys. Lett. 1990, 172, 398404. 
SYNOPSIS TOC: Tetraalkylarsonium cations, a novel member of the -onium (ammonium, phosphonium and sulfonium) family of OSDA for the synthesis of zeolites, are presented for the first time. These cations allowed synthesizing the zeolite ZSM-5 as well as served as probes for the study of the zeolite and the occluded cation by several techniques, as powder XRD, solid-state NMR and XAS.

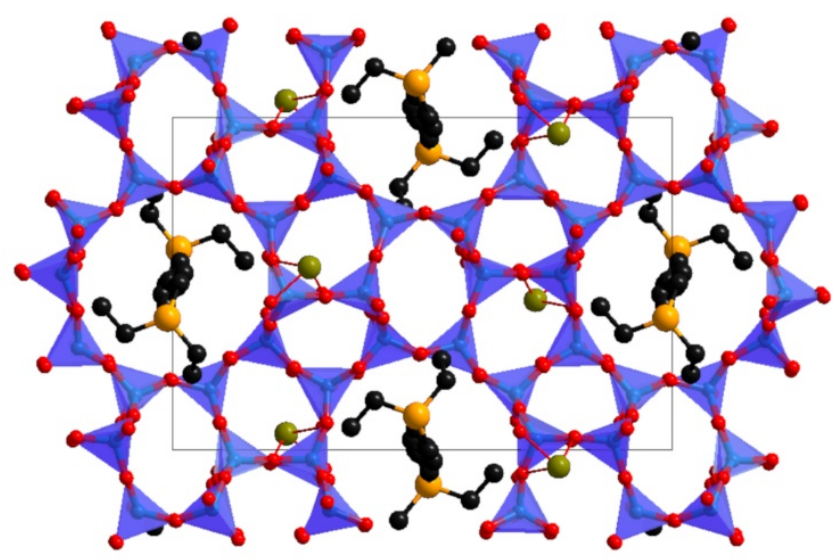

